IP Periodica Polytechnica Electrical Engineering and Computer Science

59(2), pp. 36-42, 2015

DOI: $10.3311 /$ PPee. 8165

Creative Commons Attribution (i)

RESEARCH ARTICLE

\section{Regenerative Cascaded Cell Inverter with Active Filter}

\author{
Balázs Farkas ${ }^{1,2 *}$, Károly Veszprémi ${ }^{2}$
}

Received 20 April 2015; accepted after revision 25 June 2015

\begin{abstract}
In the medium voltage application it is very beneficial to use cascaded inverters. Traditionally, this type of inverter is based on diode front end and bulky phase shifting transformer. Due to the demand of 4 quadrant drives and more severe grid requirement, the cascaded inverters with active front end have been started to emerge in the market. This article introduces regenerative cascade inverter topologies. After a short summary about the topologies, single-phase cell solution with NPC is analyzed in detail. Since one of the most challenging problems is the elimination of low order grid harmonic at low motor speed, the article demonstrates the origin of this harmonics. After the clarification of the harmonic generation phenomenon, a novel harmonic elimination method is introduced. Apart from the description of the new elimination concept, the proper controller structure is described as well. Finally, the performance of the novel method is verified by Matlab simulation.
\end{abstract}

\section{Keywords}

Cascaded Cell Inverter, AFE, Active filter, Harmonic

\footnotetext{
${ }^{1}$ Hyundai Technologies Center Hungary Ltd.,

H-1146 Budapest, Hermina út 22.

${ }^{2}$ Department of Electric Power Engineering,

Budapest University of Technology and Economics,

1111 Budapest, Egry J. utca 18., Hungary

"Corresponding author, e-mail: bfarkas@h-tec.hu
}

\section{Introduction}

Thanks to the development of the topologies and the semiconductor technology, the medium voltage (MV) segment of the drive industry grows very fast. There are a lot of special requirement and challenges in the field of the MV drives, e.g. high voltage, common mode motor voltage, limited du/dt stress and input current THD [1]. Due to special demands, the power electronic solutions differ from low voltage counterparts. Considering the aforementioned aspects, nowadays the Cascaded $\mathrm{H}$ Bridge inverters (CHB) are the most beneficial solution for the medium voltage applications [2]. This is due to the very high achievable voltage (e.g. $13 \mathrm{kV}$ ), high number of voltage levels at motor terminals and low input harmonics. The most prevalent CHB topologies are based on the Diode Front End (DFE) cell. Because of DFE, this kind of solutions can only operate in 2 quadrant mode. Other drawback of these topologies is the bulky phase shifting transformer, which is required in order to reduce THD of grid current.

Owing to the fact that 4 quadrant operation, low THD (see Table 1) and high level of grid distortion immunity are common requirements in the $\mathrm{MV}$ applications, the $\mathrm{CHB}$ with Active Front End (AFE) seems to be very attractive. Therefore, a lot of new topologies, which are based on the cell with regeneration capability, have been emerged in the last decade. Last year one of these solutions has been launched in the market [3].

Table 1 Harmonic current limits

\begin{tabular}{cccccccccc}
\hline $\begin{array}{c}\text { Harmonics } \\
\text { Order }\end{array}$ & $5^{\text {th }}$ & $7^{\text {th }}$ & $11^{\text {th }}$ & $13^{\text {th }}$ & $17^{\text {th }}$ & $19^{\text {th }}$ & $23^{\text {rd }}$ & $25^{\text {th }}$ \\
\hline $\begin{array}{c}\text { IEEE 519 } \\
(1992) \%\end{array}$ & 4 & 4 & 2 & 2 & 1.5 & 1.5 & 0.6 & 0.6 \\
\hline
\end{tabular}

Nowadays, regenerative cell inverters are extensively investigated and published. Article [4] introduces H Bridge based regenerative topologies. Matrix inverter based solutions are not only mentioned in the literature [5], but Yaskawa has started to produce this kind of cell inverter [6].

The most publications regarding regenerative CHB inverters focus on cost reduction with simpler cell solutions, on the 
harmonic analysis and elimination. In article [7] there is a detailed analysis of the input harmonic in the case of regenerative CHB with single phase cell. Another article recommends a transformer configuration in order to eliminate low order harmonics [8]. Authors of article [9] describe a control scheme to reduce capacitor load in the case of regenerative $\mathrm{CHB}$ with three phase cell. Due to the fact that main cost driver in inverters is the semiconductor, article [10] and [11] deal with the possibility of switch number reduction.

Active filter based harmonic elimination methods have not been published yet in the literature. This article introduces a novel control method so as to eliminate low order harmonics from grid current at low motor speed.

First of all, the article shortly introduces CHB solutions. The third section of the article focuses on the generation of harmonics with low and high frequency. Harmonic elimination with transformer configuration is introduced in Fig. 4. Since the applicability of this solution is very restricted by the cell number, the novel harmonic elimination method is described in the fourth section. The performance of novel harmonic elimination technique is verified by Matlab simulation in the last section.

\section{Regenerative Topologies}

There are a lot of opportunities to create AFE cells. Grid interface can be single or three phase and the rectifier topology can be Half-bridge, H-Bridge or NPC (Fig. 1) [4,10,11].

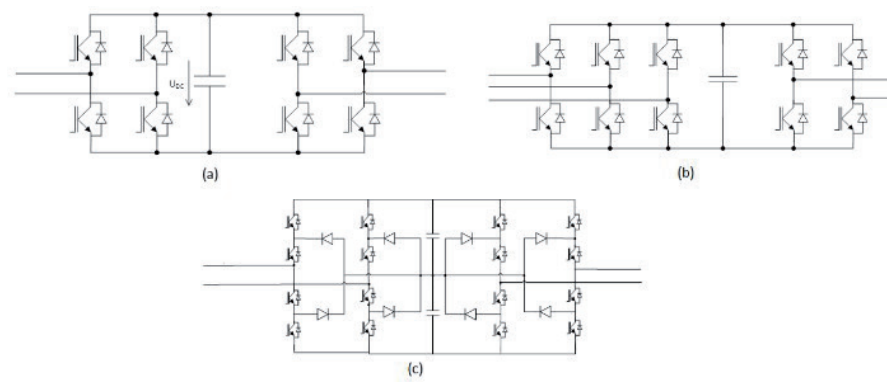

Fig. 1 Topology of the regenerative cells, (a) single-phase supply, (b) three-phase supply, (c) NPC based single-phase cell

Single-phase grid interface requires less number of switch and transformer winding than the three-phase version. In the case of single-phase supply, the motor harmonics can be observed in the grid current at low motor speed, therefore during controller design their elimination has to be considered.

The main advantage of the NPC based cell is higher achievable number of voltage levels and cell voltage. The latter is very beneficial in the MV application. NPC makes it possible to double cell voltage compared to H-Bridge. Thanks to it, the cell number and system complexity can be reduced. However, the control complexity is increased as a result of capacitor voltage balancing. Inverter with single- phase NPC cell is analysed in the following sections.

\section{Harmonics}

In this section, the harmonic generation mechanisms are introduced in the case of single-phase cell. The grid side harmonics can be divided into low- and high frequency harmonics. The origin of the low frequency harmonics is different from the CHB with DFE. In the latter case, the 6 pulse rectification is responsible for additional harmonics. As a consequence of single-phase cell- and motor supply, input power and capacitor load cannot be constant (Fig. 2). If the motor speed is very low, cell input current will be modulated by DC link voltage harmonics at high extent.

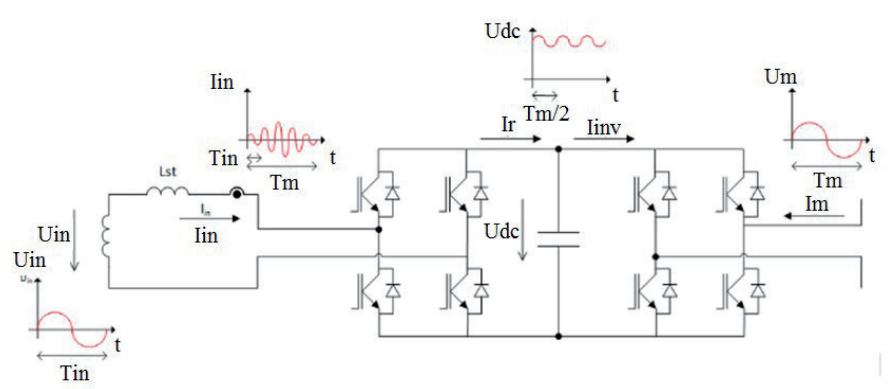

Fig. 2 Origin of the low order harmonics

Depending on the transformer configuration and cell number these harmonics can be observed in the grid current (Fig. 2).

Because of single phase load and supply, the harmonic content of the DC link voltage is relatively high. The frequencies of the main harmonics in the DC link voltage are $2 \omega_{\text {grid }}$ and $2 \omega_{\text {motor }}$. Since the crossover frequency of DC link voltage controller should be in the range of $10-70 \mathrm{~Hz}$, the DC link harmonics at low motor speed cannot be filtered. As a consequence of this, the reference signal of the current controller will be modulated by these harmonics. It means that the input current includes the harmonics of $\omega_{\text {grid }}+2 \omega_{\text {motor }}$ and $\omega_{\text {grid }}-2 \omega_{\text {motor }}$ [7]. Due to the fact that their frequency can be very close to the grid frequency, they cannot be filtered by passive elements.

In contrast to the DFE based cells, AFE generates harmonic also with switching frequency. As far as cost is concerned, using transformer leakage inductance to limit this current ripple is the best solution. However, these current harmonics can cause extra losses in the transformer and they can be observed in the grid current.

The elimination of these current harmonics from grid current and the limitation of transformer losses are crucial point of the design. Current harmonics with switching frequency can be effectively attenuated by symmetric interleave control of the AFEs supplied from the same transformer phase. If the phase shift between the carriers of these AFEs is $360 / n$, where $n$ is the cell number pro phase, the harmonics can effectively cancel each other so the high frequency harmonic content of grid current can be reduced. Total cancellation cannot be achieved. It can be explained by the AFEs output voltage distribution during 
one switching cycle. If duty is different from 0.5 for some interval of the switching period, the output voltages of two cells are the same and that is why current ripple is the same as well.

In addition, in the practical cases, the performance of this type of elimination is deteriorated by some second order effect (see Fig. 3). The most important asymmetry is tolerance of leakage inductances.
$L_{s t 1}=L_{s t 2}$

(a)

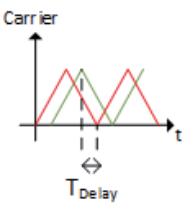

(d)

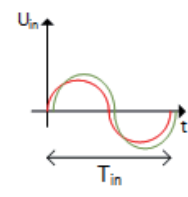

Different Current Reference

(b)

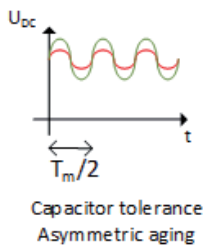

(e)

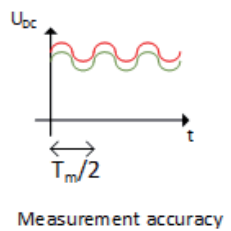

(c)

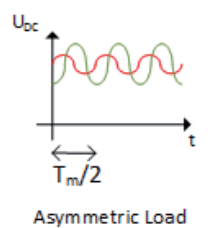

(f)
Fig. 3 Origin of the cell input current ripple deviation.

Another important source of current ripple deviation is the different reference value of the current controller. It is the case, if the cell at different motor phases is supplied from the same transformer phases. As a result of this, the voltage-time area of the leakage inductances can be different. Differences between DC link voltage and synchronization delay cause additional asymmetry.

Grid current harmonics with motor frequency can be easily eliminated if the cell number pro phase can be divided by 3 . In this case, cells from different motor phases have to be supplied from the same transformer phase and motor harmonics cancel each other (Fig. 4).

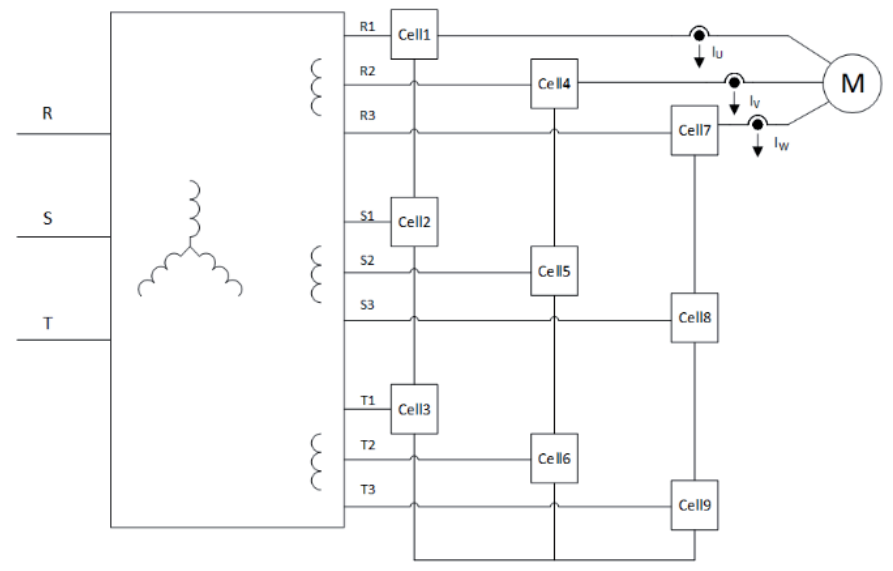

Fig. 4 Cell transformer connection configuration if the number of cell can be divided by 3 .

\section{Novel harmonic elimination method}

Aforementioned transformer based harmonic elimination method can be applied only in special cases. In this section, novel active filter based solution is introduced, which can used in more general cases. The basic concept is using some cells of the inverter as active filter to eliminate the harmonics related to motor operation. The active filter can effectively attenuate motor harmonics from the grid current at low motor speed.

Motor EMF is much lower than its nominal value at low motor speed. Therefore, less number of cells can supply the motor than at nominal speed. The unused cell injects harmonics to the grid in order to eliminate the harmonics of the other motor supplying cells. Since active filter cell does not contribute to supply the motor, the switching frequency multiplication effect at the motor side is reduced. The control method, which uses the active filter cell not only for grid harmonic injection, but also to reduce motor harmonics, is a promising future research topic. Current method uses the motor side bridge only for bypass of the cell from the motor side.

The simplest way to control the active filter is using the output of DC link voltage controller of the motor supplying cells as feedforward.

\subsection{Controller of motor supplying cell}

This controller can be divided into two main parts: motor side inverter modulator and AFE controller. Motor side modulator receives reference voltage from master controller (Um_ref). It has not got any critical role in the current harmonic elimination method. The modulator has got capacitor voltage balancing responsibility as well.

AFE controller consists of three main parts: DC link voltage controller, cell input current controller and modulator (Fig. 5).

DC link voltage controller has to stabilize DC link voltage (UDC_ref). Crossover frequency of this controller should be in the range of $10-70 \mathrm{~Hz}$, therefore the output of the controller includes the harmonics with doubled motor frequency. It is multiplied by a sine function with grid frequency $(\mathrm{Ug} /|\mathrm{Ug}|)$ to create reference signal of the current controller. It is worth applying some feedforward from the motor load to improve dynamic performance of DC link controller (FFWDmot).

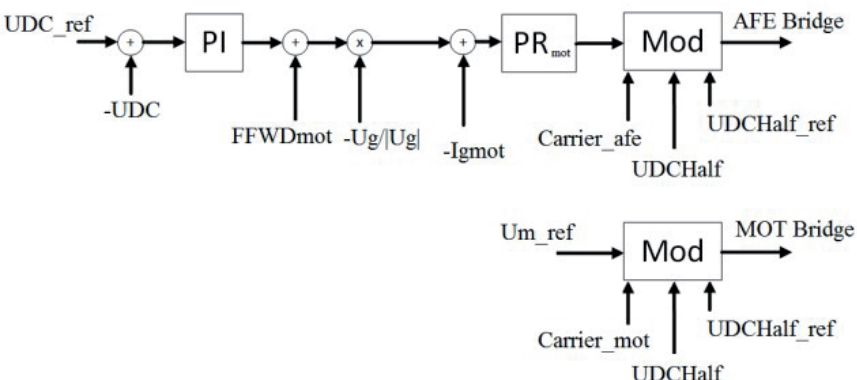

Fig. 5 The controller of the motor supplying cell. 
The reference signal of the current controller is an amplitude modulated signal because of the multiplication and it includes the following main harmonics: $\omega_{\text {grid }} \pm 2 \omega_{\text {motor }}$. This signal can be used as feedforward for active filter if the fundamental component is removed. The easiest way to remove the fundamental frequency is when output signal of DC voltage controller is filtered by high pass filter and then multiplied with grid frequency sine function in the opposite phase.

\subsection{Controller of active filter cell}

There are three differences between the active filter cell controller and the motor supplying cell controller: external feedforward signal, harmonic compensator and voltage controller role.

The current controller has two parts (Fig. 6). The first is feedforward which uses external feedforward signal (FFWDactf) as reference. This block mimics the current controller of the motor supplying cell. The output signal of DC link controller in motor supplying cell can be used as feedforward signal after the DC component is removed. The second part is the harmonic compensator (Ractf). It consists of several resonant controllers which are tuned to relevant grid current harmonics. Feedback signal is the sum of input currents of the active filter (Igactf) and motor supplying cells (Igmot) (Fig. 6).

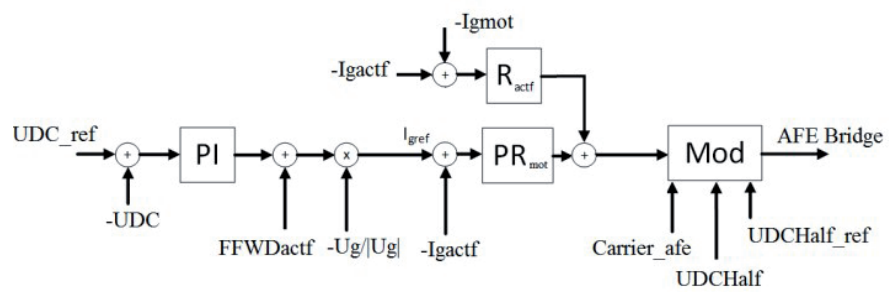

Fig. 6 The controller of the active filter cell.

Since active filter does not supply the motor, there is no real power flow so the load of DC link capacitors is determined only by harmonics. This load is much lower than the load of the motor supplying cell. Therefore, the DC link voltage controller should compensate only this kind of load. The signal of DC link voltage controller is added to the feedforward signal to create the reference signal for current controller.

\subsection{Elimination of the high frequency current harmonis}

Another important aspect of the active filter control is high frequency current ripple. With interleave control of the AFE in motor supplying cell and in active filter, it is possible to reduce the transformer ripple current like in normal cases. The efficacy of the harmonic reduction is smaller than it was in two symmetric cells.

\section{Simulation}

CHB with $3 \times 2$ cells is used for the verification of the control scheme. The motor nominal voltage is $3.3 \mathrm{kV}$ and the inverter is supplied from $3.3 \mathrm{kV}$ grid. The load profile is chosen to constant torque. It means that motor current equals its nominal value, but the motor speed is only $10 \%$ of the nominal value.

\subsection{Parameters}

Table 2 Transformer flux harmonics

\begin{tabular}{|c|c|}
\hline Name & Value \\
\hline Grid Nominal Voltage & $3.3 \mathrm{kV}_{\mathrm{RMS}}$ \\
\hline Grid Nominal Input Current & $189 \mathrm{~A}_{\mathrm{RMS}}$ \\
\hline Transformer Turn Ratio & $2: 1$ \\
\hline Number of Transformer Secondary & $3 \times 2$ \\
\hline Phase Shifting & No \\
\hline Grid side transformer leakage inductance & $3 \% ; 1000 \mathrm{uH}$ \\
\hline Inverter side transf. leakage inductance & $7 \% ; 1000 \mathrm{uH}$ \\
\hline Transformer Magnetizing Inductance & $5600 \% ; 0.8 \mathrm{H}$ \\
\hline Cell Nominal Power & $200 \mathrm{~kW}$ \\
\hline Cell Nominal Input Current & $210 \mathrm{~A}_{\mathrm{RMS}}$ \\
\hline Cell DC link Capacitance & $2.25 \mathrm{mF}$ \\
\hline Cell DC link Voltage & $1600 \mathrm{~V}$ \\
\hline AFE Switching Frequency & $3 \mathrm{kHz}$ \\
\hline Motor Side Inverter Sw. frequency & $1.5 \mathrm{kHz}$ \\
\hline Motor Nominal App. Power & 1.2 MVA \\
\hline Motor Nominal Voltage & $3.3 \mathrm{kV}_{\mathrm{RMS}}$ \\
\hline Motor $\cos (\phi)$ & 0.9 \\
\hline Motor Nominal Frequency & $50 \mathrm{~Hz}$ \\
\hline Motor Nominal Current & $214 \mathrm{~A}_{\mathrm{RMS}}$ \\
\hline Motor Leakage Inductance & $12 \% ; 4.2 \mathrm{mH}$ \\
\hline Motor Frequency & $5 \mathrm{~Hz}$ \\
\hline Motor Voltage & $330 \mathrm{~V}_{\mathrm{RMS}}$ \\
\hline Motor App. Power & $120 \mathrm{kVA}$ \\
\hline Motor Current & $214 \mathrm{~A}_{\mathrm{RMS}}$ \\
\hline
\end{tabular}




\subsection{Simulation Results}
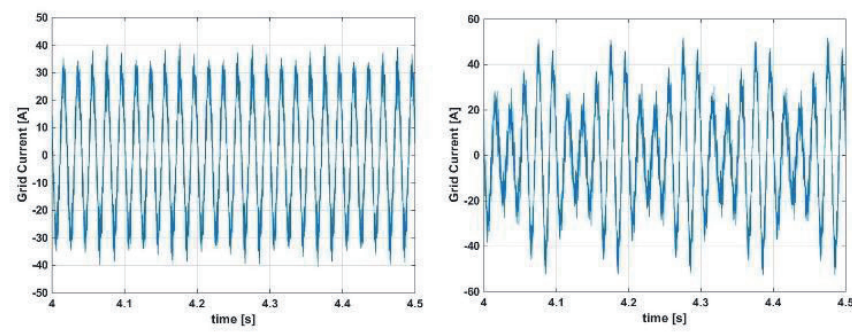

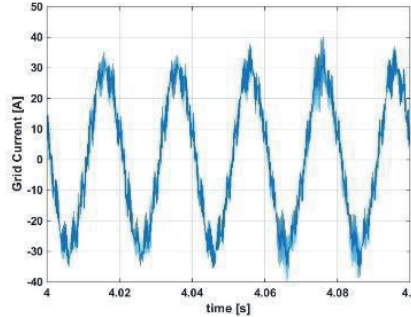

(a)

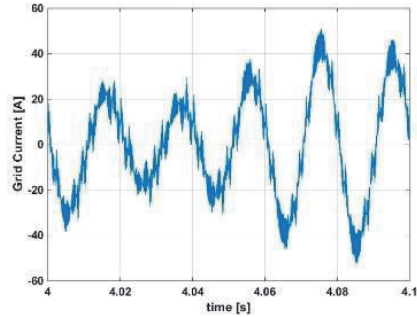

(b)
Fig. 7 Grid Current with harmonic elimination (a) and without harmonic elimination (b)

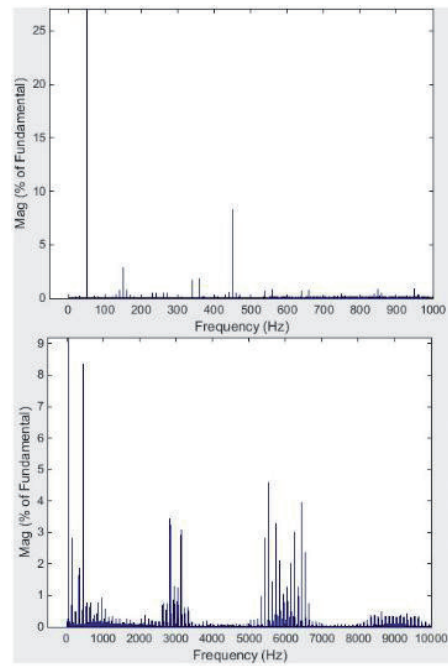

(a)
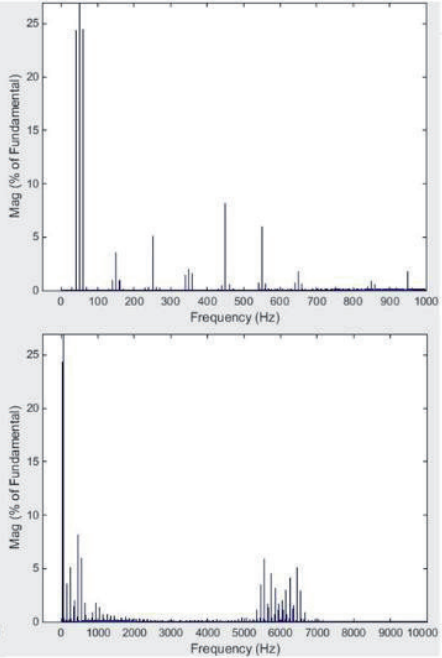

(b)

Fig. 8 Harmonics of the grid current with harmonic elimination (a) and without harmonic elimination (b)

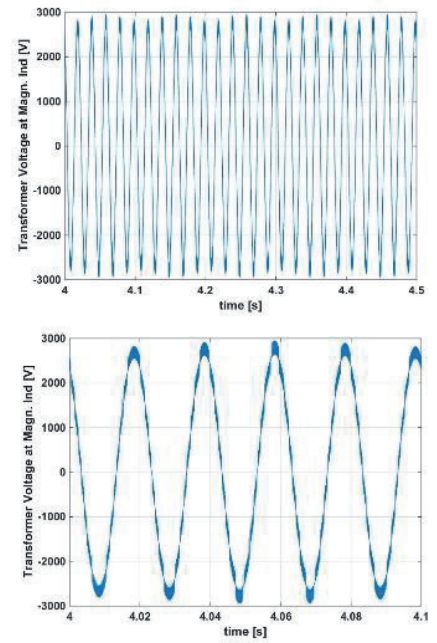

(a)
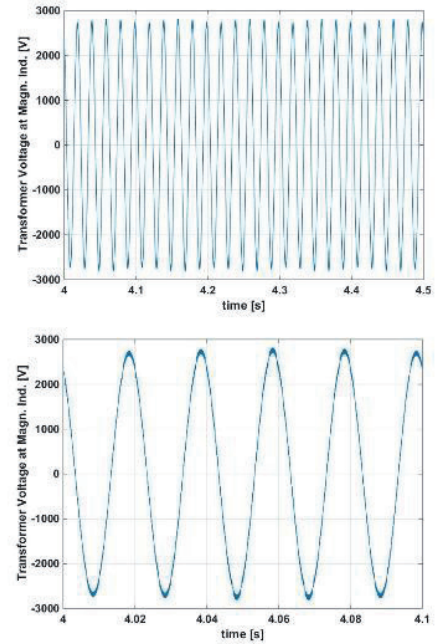

(b)

Fig. 9 Transformer voltage with harmonic elimination (a) and without harmonic elimination (b)

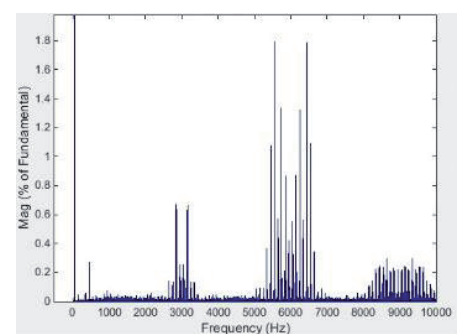

(a)

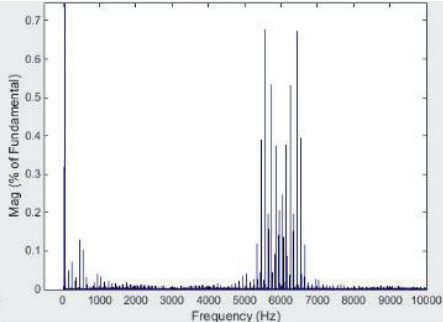

(b)
Fig. 10 Harmonics of Transformer voltage with harmonic elimination (a) and without harmonic elimination (b)

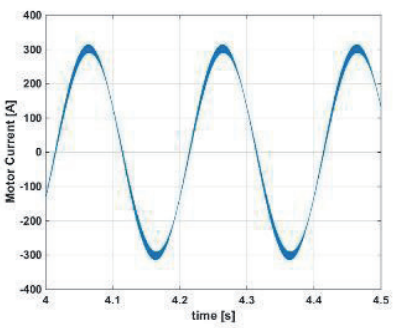

(a)

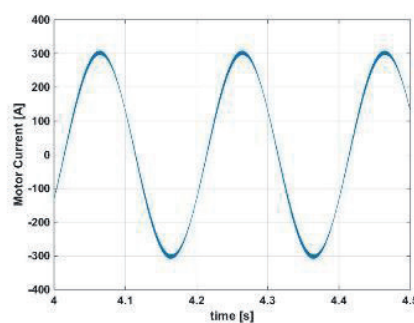

(b)
Fig. 11 Motor current with harmonic elimination (a) and without harmonic elimination (b)

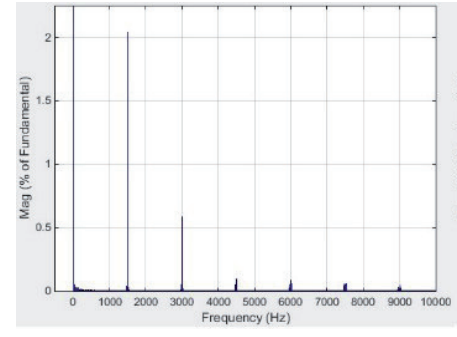

(a)

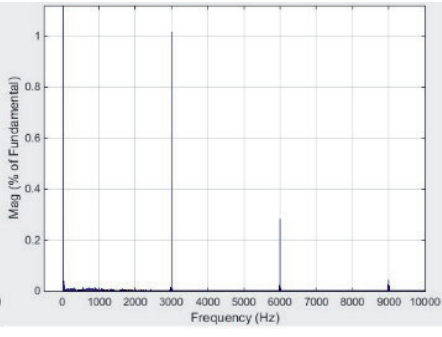

(b)
Fig. 12 Harmonics of the motor current with harmonic elimination (a) and without harmonic elimination (b)

\subsection{Analysis}

The performance of the recommended control scheme is investigated based on the following criteria:

- Reduction of the main current harmonics $\left(\omega_{\text {grid }}+2 \omega_{\text {motor }}\right.$ and $\left.\omega_{\text {grid }}-2 \omega_{\text {motor }}\right)$

- Reduction of the high frequency harmonics in the grid current

- Requirements of the IEEE 519

- Transformer voltage harmonics

- Motor Current Harmonics

Figure 8.b shows the spectrum of the grid current if no harmonic elimination method is used. According to previous expectation, the harmonics of $\omega_{\text {grid }}+2 \omega_{\text {motor }}$ and $\omega_{\text {grid }}+2 \omega_{\text {motor }}$ are the most prevalent in the spectrum. Their magnitude is approximately $24 \%$. The effect of the recommended control scheme can be seen in Fig. 8.a. The aforementioned two harmonics are effectively removed from the spectrum. Apart from low order 
harmonics, there are a lot of high frequency current harmonics in the spectrum in both cases. If both cells supply the motor and they operate in the same load condition, the carrier shift technique can effectively attenuate switching frequency harmonics. Around $3 \mathrm{kHz}$ there is no harmonic in the spectrum (see Fig. 8.b). However, there are some harmonics around doubled switching frequency with a magnitude of $4-5 \%$. It meets previous expectations (Section 3). If one of the cells is used as active filter, the harmonics at the switching frequency can be observed (Fig. 8.a), their magnitude is about 2-3\%. Apart from them, the magnitude of harmonics around the doubled switching frequency remains approximately the same as in symmetric case. The differences between the harmonics at switching frequency are the consequence of different operating conditions of two cells.

If no harmonic elimination technique is applied, the low order harmonics do not meet the requirements of the IEEE 519. In case of the active filter, all harmonics up to order 25 in the grid current are far below the IEEE 519 limits. The harmonics around the switching frequency have relative high magnitude; however, they can be easily filtered.

The current harmonics with high frequency generate extra losses in the primary winding of the transformer thanks to the skin and proximity effect. The extent of the current harmonics generated losses is much higher at the secondary winding, because of the fact that harmonic cancellation is applied only in the primary side. The calculation of these extra losses is beyond the scope of this article, because they depend on lots of factors, e.g. winding geometry, transformer construction etc.

Fig. 9 and Fig. 10 show the transformer voltage at the magnetizing inductance. Because of AFE operation, the flux of the transformer includes some harmonics which generate extra core losses. It would be important to decide whether the voltage harmonic level can be acceptable or not. The THD of the most prevalent voltage harmonics at magnetizing inductance is approximately $1.13 \%$ at $3 \mathrm{kHz}$ and $8.52 \%$ at $6 \mathrm{kHz}$. Based on these figures, magnitude of the transformer flux harmonics can be estimated. Effect of the magnetizing inductance has to be considered in order to calculate the flux harmonics:

$$
\Psi_{n}=U_{n} / n
$$

Table 3 Transformer flux harmonics

\begin{tabular}{ccc}
\hline Voltage Harmonics & Frequency & Flux Harmonics \\
\hline $1,13 \%$ & $3 \mathrm{kHz}$ & $0,018 \%$ \\
$8,52 \%$ & $6 \mathrm{kHz}$ & $0,071 \%$ \\
\hline
\end{tabular}

Steinmetz equation can be used to estimate the ratio of fund amental and harmonic core losses:

$$
P_{\text {core }}=C \times f^{\alpha} B^{\beta}
$$

Typically $\alpha$ is approximately 3 and $\beta$ is 2 . Ratio of fundamental and harmonic core losses:

$$
\begin{aligned}
& P_{\text {coreh } 3 k H z} / P_{\text {coref }}=(3000 / 50)^{3} \times 0,00018^{2}=7 \% \\
& P_{\text {coreh } 6 k H z} / P_{\text {coref }}=(6000 / 50)^{3} \times 0,00071^{2}=87 \%
\end{aligned}
$$

Supposing that copper losses and core losses at fundamental frequency are almost the same, total losses of the transformer is increased by $47 \%$. Since $6 \mathrm{kHz}$ voltage harmonics are the main loss contributors, transformer additional core losses are almost independent of the active filter operation. Additional winding losses are not included in this calculation.

Since one of the cells is used as active filter, the harmonic content of the motor voltage and current are increased compared to the normal operation. Thanks to low speed, motor EMF is much lower than its nominal value. Therefore, the voltage-area at motor leakage inductance is relatively small. Due to this fact, motor current includes negligible harmonics: around $2 \%$ (Fig 12.a) in case of active filter configuration and $1 \%$ in the normal case (Fig 12.b). The switching frequency multiplication effect disappears if one of the cells operates as active filter. It means that active filter configuration has not got strong effect on motor current.

\section{Conclusion}

Relevant regenerative CHB topologies are shortly summarized in the first part of the article. Not only the topologies, but also the origin of the low and high frequency harmonics are introduced. In the second part of the article, novel control scheme of the inverter is introduced. The concept is based on the use of some cells as active filter in order to compensate low order grid harmonics. This solution is deeply analyzed and the controller structure is described. Finally, the performance of the solution is verified by Matlab simulation.

\section{Acknowledgement}

The project presented in this article is supported by Hyundai Technology Center Hungary Ltd. and Budapest University of Technology and Economics Department of Electric Power Engineering.

\section{References}

[1] Abu-Rub, H., Holtz, J., Rodriguez, J. "Medium- Voltage Multilevel Converters - State of the Art, Challenges, and Requirements in Industrial Applications." IEEE Transactions on Industrial Electronics. 57 (8). pp. 2581-2596. 2010. DOI: 10.1109/tie.2010.2043039

[2] Malinowski, M., Gopakumar, K., Rodríguez, J. "A Survey on Cascaded Multilevel Inverters." IEEE Transactions on Industrial Electronics. 57 (7). pp. 2197-2206. 2010. DOI: 10.1109/tie.2009.2030767

[3] TMEIC "TMdrive-MVe2 Series Product Application Guide." 2014. 
[4] Rodriguez, J., Pontt, J., Silva, E., Espinoza, J., Perez, M. "Topologies for Regenerative Cascaded Multilevel Inverters." In: Power Electronics Specialist Conference, 2003. PESC '03. 2003 IEEE 34th Annual. Vol. 2. pp. 519-524. 15-19 June 2003. DOI: 10.1109/pesc.2003.1218109

[5] Kouro, S., Malinowski, M., Rodriguez, J. "Recent Advances and Industrial Applications of Multilevel Converters." IEEE Transactions on Industrial Electronics. 57 (8). pp. 2553-2580. 2010.

DOI: $10.1109 /$ tie.2010.2049719

[6] Yaskawa - FSDrive-MV1S, Matrix inverter

[7] Rodriguez, J., Lezana, P., Espinoza, J., Perez, M. "Input Current Harmonics in a Regenerative Multi-Cell Inverter with Single Phase Active Rectifier." In: IECON'02 [Industrial Electronics Society, IEEE 2002 28th Annual Conference of the]. Vol. 2. pp. 932-937. 5-8 Nov. 2002. DOI: 10.1109/iecon.2002.1185397

[8] Rodríguez, J., Morán, L., Gonzalez, A., Silva, C. "High-Voltage Multilevel Converter with Regeneration Capability." IEEE Transactions on Industrial Electronics. 49 (4). pp. 839-846. 2002.

DOI: $10.1109 /$ tie.2002.801238
[9] Perez, M., Rodriguez, J., Lezana, P. "Regenerative Medium-Voltage AC Drive based on a Multi-Cell Arrangement with Minimum Energy Storage Requirements.” IEEE Transactions on Industrial Electronics. 52. pp. 171-180. 2005. DOI: 10.1109/tie.2004.841095

[10] Lezana, P., Rodríguez, J., Rojas, D., Pontt, J. "Novel Cell based on Reduced Single-phase Active Front End for Multicell Converters." Industrial Electronics Society, 2005. IECON 2005. 31st Annual Conference of IEEE. pp. 733-738. 6-6 Nov. 2005. DOI: 10.1109/iecon.2005.1568995

[11] Lezana, P., Rodríguez, J., Oyarzún, D. A. "Cascaded Multilevel Inverter with Regeneration Capability and Reduced Number of Switches." IEEE Transactions on Industrial Electronics. 55 (3). pp. 1059-1066. 2008. DOI: 10.1109/tie.2008.917095 